\title{
PID and Adaptive Controllers for a Transportation Mobile Robot with Fork-Type Lifter
}

Nguyen Van Vui, Tran Huu Luat, and Yong-Tae Kim

Department of Electrical, Electronic and Control Engineering, Hankyong National University, Anseong, Korea
Received: Sep. 18, 2016

Revised : Sep. 23, 2016

Accepted: Sep. 24, 2016

Correspondence to: Yong-Tae Kim

(E-mail: ytkim@hknu.ac.kr)

(CThe Korean Institute of Intelligent Systems

(C)This is an Open Access article distributed under the terms of the Creative Commons Attribution Non-Commercial License (http://creativecommons.org/licenses/ by-nc/3.0// which permits unrestricted noncommercial use, distribution, and reproduction in any medium, provided the original work is properly cited.

\begin{abstract}
This paper proposes a new controller design method for a fork-type lifter (FTL) of a transportation mobile robot. The transportation robot needs to pick up a package from a stack on a storage shelf and move on by a planned path in a logistics center environment. The position of the storage shelf is recognized by reading a QR code on the floor, and using this position, the robot can move to reach the storage shelf and pick up the package. PID controllers and an adaptive controller are designed to control the velocity of two wheels and the position of the FTL. An adaptive controller for the lifter is designed to elevate up and down on a slideway to the correct height position of the package on the stack of the storage shelf. The simulation results show that the PID controllers can respond smoothly to the desired angular velocity and the adaptive controller can adapt quickly and correctly to the desired height.
\end{abstract}

Keywords: Fork-type lifter system, Transportation mobile robot, Kalman filter, Adaptive controller, PID controller

\section{Introduction}

Todays logistics centers are seeing increased demands to adapt for goods transportation worldwide. Storage shelves, located everywhere in the logistics center, are typically used to store goods packages. A transportation robot deployed in the logistics center needs must identify the exact position of a storage shelf on a planned path [1] and the position of a package in a stack on the storage shelf. Therefore, a fork-type lifter (FTL) of the robot should be designed to elevate up and down on a slideway and it must be moved out to pick up the package from the stack on the storage shelf. The slideway generates a sliding surface between the FTL and its frame and the FTL rises along the way when it is moving to the exact height.

In order to solve this problem, a QR code is put on the floor at the position of the storage shelf and the robot reads it to find the location [2]. Paths are defined by reading the QR codes with the robots camera. In addition, the position of the package on the storage shelf can be calculated based on the height of the storage shelf. The storage shelf can has several stacks and each stack is designated with the correct height and managed by a server. The server manages the location of all packages for planning the path. The robot communicates continuously with the server to determine the location of package when it is moving [2].

In addition, the robot can estimate its position on the path by using encoder sensors on the 


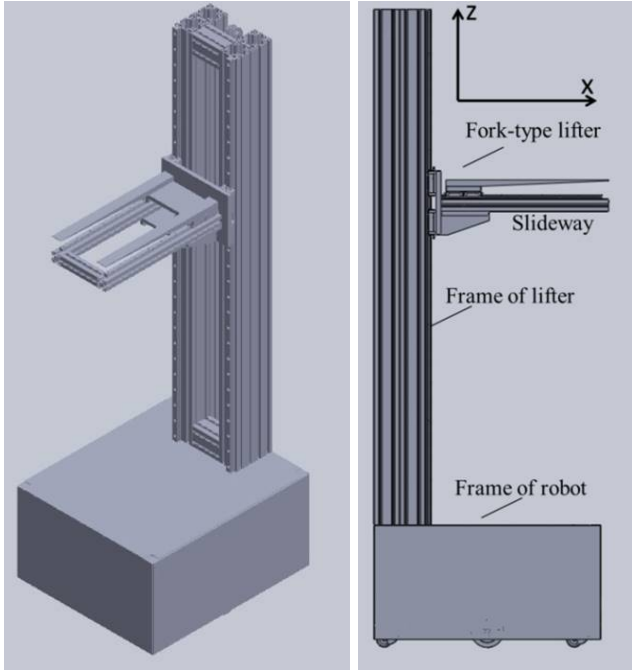

Figure 1. Configuration of the transportation mobile robot with FTL.

motors in two wheels and the FTL. A Kalman filter is used to decrease the noise of encoder sensors [3], and the corrected data from the encoder sensors helps the robot calculate its exact location on the path.

In this paper, PID controllers were designed to control the velocity of two wheels. Furthermore, a sliding surface was defined to develop an adaptive controller for the FTL. The control gains of this controller are self-tuned by using an adaptive algorithm. The proposed method shows that the robot reaches and picks up the package more correctly and smoothly.

This paper is organized as follows. In Section 2, the mechanical configuration of the FTL system is presented. In Section 3, we present a kinematic and dynamic model of the transportation mobile robot with FTL. In Section 4, we present the design of an adaptive controller for the FTL. In Section 5, the design of the overall controller of the system is discussed. In Section 6, the simulation results of the proposed method are presented.

\section{Design of the FTL System}

The FTL system includes two main parts: the lifter and the forktype element (FTE). The lifter can be elevated up and down on the slideway. The lifter motor is fixed on the frame of the robot and transfers its torque to become a transitivity force on the lifter along $\mathrm{z}$-axis path as shown in Figure 1.

The FTE shown in Figure 2 is designed the same as the lifter but it moves following the $\mathrm{x}$-axis path. Its direction starts from the side of the lifter's frame to the position of the storage shelf. The FTE moves back and forth to pick up the package on the stack on the storage shelf.

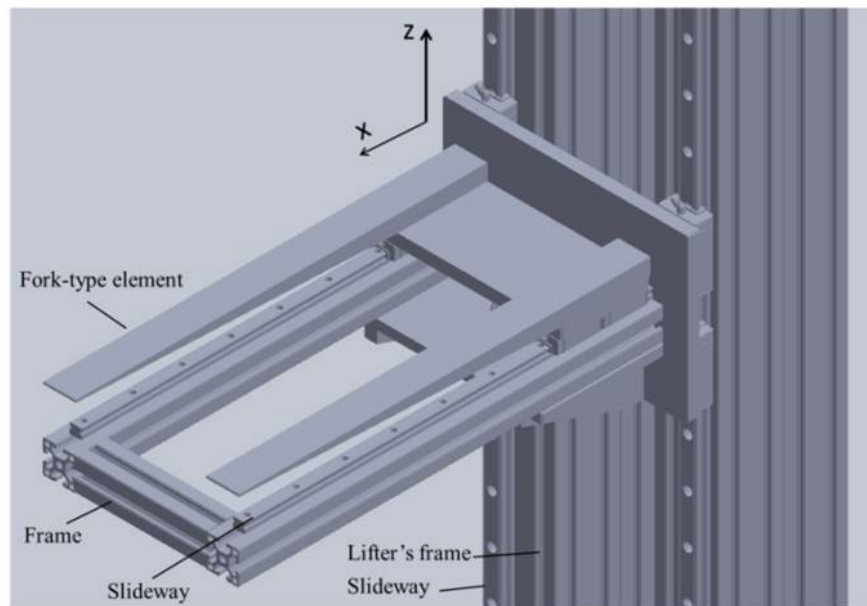

Figure 2. Design of FTE.

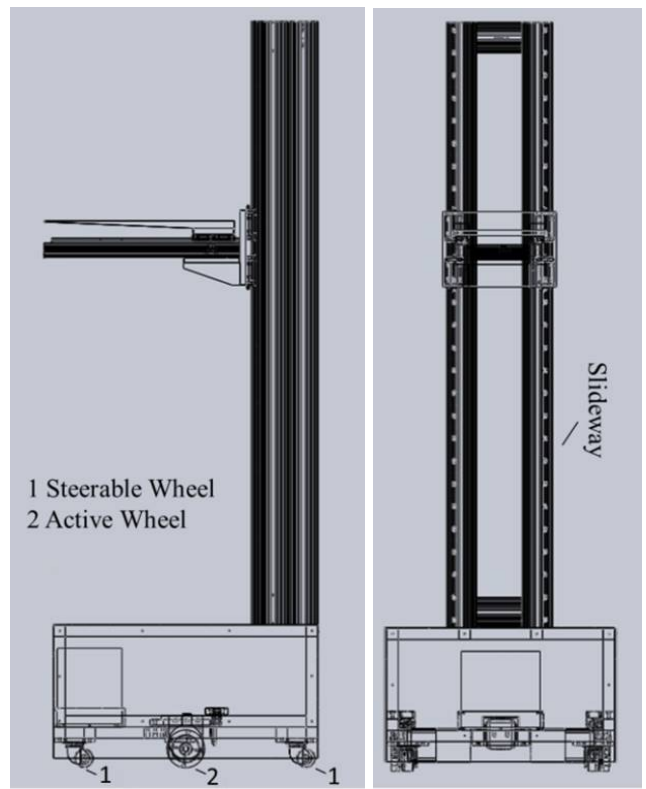

Figure 3. Mobile platform design of the transportation robot.

The side of the FTE and the side of the robots frame are same. This configuration can help the length of the FTEs frame compensate the length of the robots frame when the robot is reaching the storage shelf. The slideway is designed to generate a sliding surface between the lifters frame and the lifter, and between the FTEs frame and the FTE. This design method can make the FTL moves more easily and smoothly.

The mobile platform of the transportation robot is designed as shown in Figure 3. It is designed based on non-holonomic type of mobile robot [4]. It includes two active wheels and four steerable wheels.

We designed the robots frame to move on the planned path 


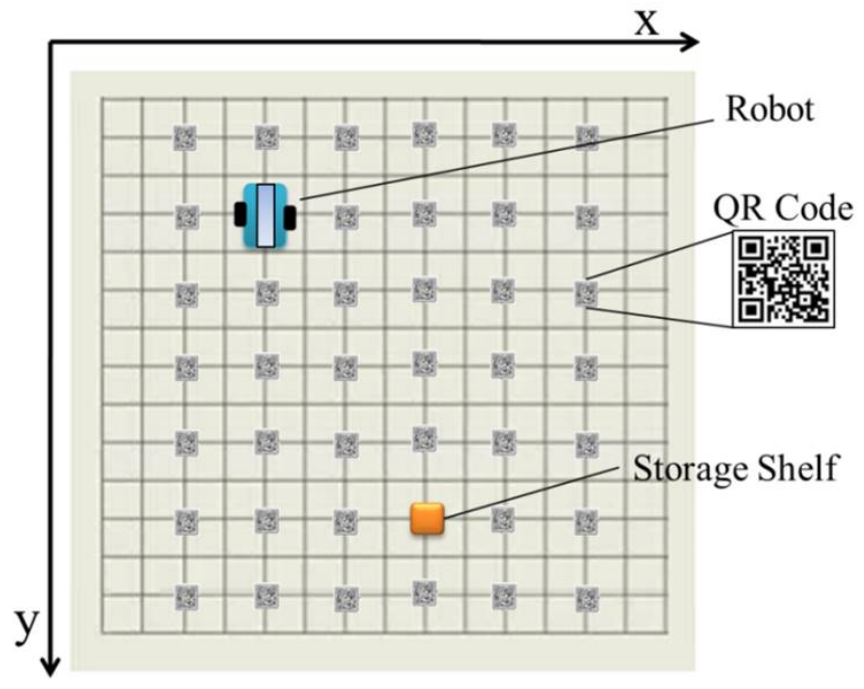

Figure 4. QR-code map on the warehouses floor.

Table 1. Parameters of the FTL system

\begin{tabular}{cc}
\hline Contents & Values (mm) \\
\hline Width of robot's frame (d) & 600.0 \\
\hline Length of robots frame (L) & 700.0 \\
\hline Radius of steerable wheel & 24.4 \\
\hline Radius of active wheel (R) & 50.0 \\
\hline Height of lifters frame & $1,596.0$ \\
\hline Length of lifter's frame & 300.0 \\
Width of FTE & 196.0 \\
\hline Length of FTE & 503.5 \\
\hline
\end{tabular}

easily. We find a path defined based on a QR-code map on the warehouse floor as shown in Figure 4. QR-codes are placed on the grid points of the floor with a fixed distance. This map is used by the robot to move from the start point to the storage shelf correctly.

Especially, the design of the robots frame should be focused on solving the weight balance between frame of the robot and the FTL system. When the FTL elevates up and moves out to pick up the package on the storage shelf, the barycenter of the robot moves, following the FTE. Therefore, the robots frame shown in Figure 2 should be designed to keep a balance between total of the weight of the FTL system and the package weight. The parameters of the FTL system are presented in Table 1.

\section{Kinematic and Dynamic Models of the Trans- portation Mobile Robot with FTL}

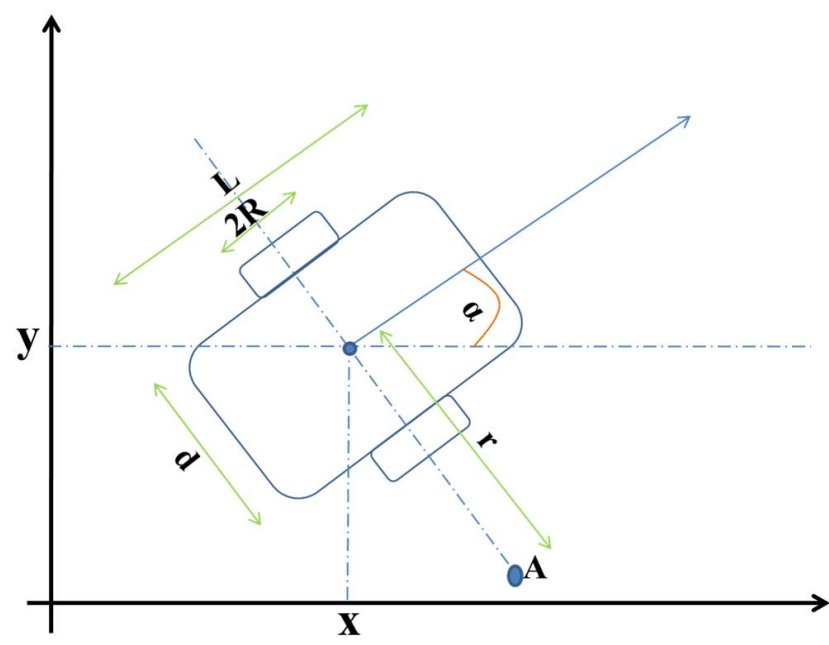

Figure 5. Kinematic model of a two-wheeled mobile robot.

\subsection{Kinematic Model of the Mobile Robot}

The transportation mobile robot is designed to move by two active wheels on the floor [5]. The kinematic model of a wheeled mobile robot is configured as shown in Figure 5 [6-14].

$r$ is the instantaneous curvature radius of the robot trajectory, $d$ is distance between two wheels, $R$ is wheel radius, and $\alpha$ is angle between the robot's orientation and x-axis path.

This robot configuration uses independent linear velocities, $v_{R}$ for the right wheel and $v_{L}$ for the left wheel to move to a desired point $(x, y)$ and a desired orientation $\alpha$. The linear velocity of a wheel is directly proportional to its angular velocity.

The relation between the linear velocity $v(t)$ and angular velocity $\omega(t)$ of the robot depends on the linear velocities of the left and right wheel.

The angular velocity of the robot is calculated by the following equations [7].

$$
\begin{aligned}
& \omega=\frac{v_{R}}{R-\frac{d}{2}}, \\
& \omega=\frac{v_{L}}{R+\frac{d}{2}} .
\end{aligned}
$$

From Eq. (1) and Eq. (2), the angular velocity can be derived as

$$
\omega=\frac{\left(v_{L}-v_{R}\right)}{d}
$$

Then, the linear velocity $v$ is obtained as the following equation.

$$
v=\omega R=\frac{\left(v_{L}+v_{R}\right)}{2}
$$




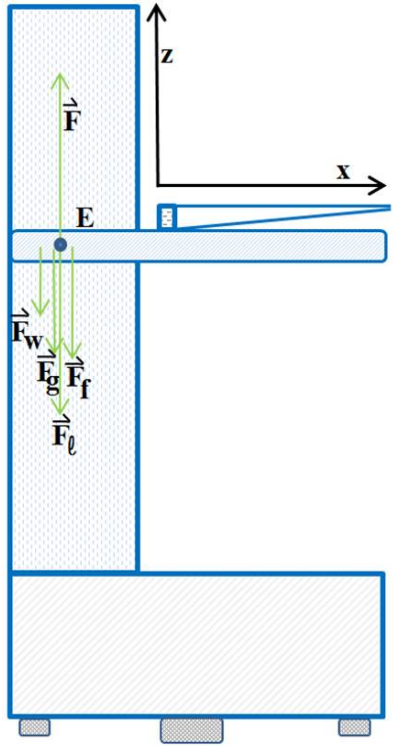

Figure 6. Motion analysis of the FTL.

In addition, the robots position is calculated based on the linear velocity $v$ and the angular velocity as belows.

$$
\left[\begin{array}{c}
\dot{x} \\
\dot{y} \\
\dot{\alpha}
\end{array}\right]=\left[\begin{array}{cc}
\cos \alpha & 0 \\
\sin \alpha & 0 \\
0 & 1
\end{array}\right]\left[\begin{array}{l}
v \\
\omega
\end{array}\right] \text {. }
$$

From Eq. [5], the kinematic model of the mobile robot can be written as

$$
\left\{\begin{array}{l}
\dot{x} \sin \alpha-\dot{y}=0, \\
\dot{x}=v \cos \alpha, \\
\dot{y}=v \sin \alpha, \\
\dot{\alpha}=\omega .
\end{array}\right.
$$

\subsection{Dynamic Model of FTL}

In this section, we analyze the dynamic model of the FTL. The FTL moves along the z-axis path and the FTE moves along the $\mathrm{x}$-axis path.

The dynamic model can be obtained by analyzing the component of forces at point $E$ on the lifter as shown in Figure 6 . Force is generated from the motor, $F$ depend on acceleration $a$ and mass of FTE $m$. Forces such as the force of load $F_{l}$, wrinkle force $F_{w}$, force of friction $F_{f}$ and gravity force $F_{g}$ are generated conversely with the motor force $F$.

Let us consider the following motion equation,

$$
m \ddot{z}(t)=F(t)-\left[F_{l}(t)+F_{f}(t)+F_{w}(t)+F_{g}(t)\right],
$$

where $z(t)$ is position of the lifter, $F_{l}$ is force of load that depends on load along the slideway axis, $F_{f}$ is friction force that is generated when the lifter moves on the slideway, $F_{w}$ is wrinkle force that is generated when the lifter moves and $F_{g}=m g$ is gravity force.

A mathematical model of motor based on its dynamics model is represented as

$$
\begin{aligned}
& u(t)=K_{e} \dot{z}(t)+R i(t)+L \frac{d i(t)}{d t}, \\
& F(t)=K_{f} i(t),
\end{aligned}
$$

where $R$ and $L$ are the resistor and induction coil. $u(t)$ and $i(t)$ are the time-varying motor terminal voltage and armature current respectively, $F(t)$ is the transitivity force of the motor that is generated when supplying power. $K_{e}$ and $K_{f}$ are constant gains.

From Eqs. (7), (8) and (9), we have following equation

$$
\ddot{z}=\frac{1}{m}\left[-\frac{K_{e} K_{f}}{R} \dot{z}+\frac{K_{f}}{R} u(t)-\left(F_{l}+F_{f}+F_{w}+F_{g}\right)\right] .
$$

Eq. (10) can be rewritten as follows:

$$
\ddot{z}=\frac{1}{B}\left[A \dot{z}+u-\left(F_{l}^{\prime}+F_{f}^{\prime}+F_{w}^{\prime}+F_{g}^{\prime}\right)\right],
$$

where $A=-K_{e}, B=\frac{m R}{K_{f}}, F_{l}^{\prime}=\frac{R}{K_{f}} F_{l}, F_{f}^{\prime}=\frac{R}{K_{f}} F_{f}$, $F_{w}^{\prime}=\frac{R}{K_{f}} F_{w}$ and $F_{g}^{\prime}=\frac{R}{K_{f}} F_{g}$.

\section{Design of Adaptive Control of the FTL}

We consider a linear motor as a dynamic model with uncertain parameters. The uncertainties occur because of the structural properties and external disturbances. In this section we design an adaptive controller to solve this problem.

From the dynamic equation Eq. [11, we obtain a state equation by taking the state variables as $z_{1}=z, z_{2}=\dot{z}_{1}$ and $z_{3}=u$.

The nonlinear equation of system is rewritten as

$$
\dot{z}_{2}=\frac{1}{B}\left[A z_{2}+z_{3}-\left(F_{l}^{\prime}+F_{f}^{\prime}+F_{w}^{\prime}+F_{g}^{\prime}\right)\right] .
$$

The output of system, $z_{2}$ tracks to a reference signal $z_{2 d}$, we define the tracking error as follows:

$$
e(t)=z_{2}(t)-z_{2 d}(t) .
$$


The derivative of the error is given by

$$
\dot{e}(t)=\dot{z_{2}}(t)-z_{\dot{2} d}(t)
$$

where $z_{2 d}$ and $z_{2 d}$ are the desired position and velocity, respectively.

Then, we define the sliding surface given by

$$
s=\left(\frac{d}{d t}+\lambda\right) e=\dot{e}+\lambda e
$$

where $\lambda$ is a given positive constant.

An error metric $s_{\Delta}(t)$ is defined as belows.

$$
s_{\Delta}(t)=s(t)-\delta \operatorname{sat}(s(t) / \delta)
$$

where $\delta$ is a boundary layer around the sliding surface. The control law of the adaptive controller is tracking error metric $s_{\Delta}(t)$.

Then, the control input $u$ of the system is given as follows:

$$
u=-\hat{g} z_{2}-\hat{h} u_{c}+\left(\hat{k}_{1}+\hat{k}_{2}\left|z_{2}\right|\right) \operatorname{sgn}\left(s_{\Delta}\right),
$$

where $\hat{g}$ and $\hat{h}$ are the estimates from $g$ and $h$, respectively, and $\hat{k}_{0}$ represents the estimate of $k_{0} \cdot u_{c}$ is an additional controller as

$$
u_{c}=-c \dot{e}-\ddot{z}_{2}-\lambda s_{\Delta} .
$$

The adaptation law for the parameters in Eq. (17) is determined as the following equations.

$$
\begin{aligned}
& \dot{\hat{g}}=-r_{1} s_{\Delta}, \\
& \dot{\widehat{h}}=-r_{2} u_{c} s_{\Delta}, \\
& \dot{\widehat{k_{1}}}=r_{3}\left|s_{\Delta}\right|, \\
& \dot{\widehat{k_{2}}}=r_{4}|\dot{z}|\left|s_{\Delta}\right|,
\end{aligned}
$$

where $r_{1}, r_{2}, r_{3}$, and $r_{4}$ are positive constants.

The stability and convergence of this adaptive control system can be analyzed by using Lyapunov theory. We use Lyapunov function candidate $V(t)$ as

$$
V(t)=\frac{1}{2} b s^{2}+\frac{1}{2 r_{1}} \tilde{g}^{2}+\frac{1}{2 r_{2}} \tilde{h}^{2}+\frac{1}{2 r_{3}} \tilde{k}_{1}^{2}+\frac{1}{2 r_{4}}=\tilde{k}_{2}^{2},
$$

where $\tilde{a}=a-\hat{a}, \tilde{b}=b-\hat{b}, \tilde{k}_{1}=k_{1}-\widehat{k_{1}}$ and $\tilde{k}_{2}=k_{2}=\widehat{k_{2}}$.
The derivative of $V(t)$ is derived as follows:

$$
\dot{V}(t)=b \dot{s} s_{\Delta}+\frac{1}{r_{1}} \dot{\tilde{g}} \tilde{g}+\frac{1}{r_{2}} \dot{\tilde{h}} \tilde{h}+\frac{1}{r_{3}} \dot{\tilde{k}}_{1} \tilde{k}_{1}+\frac{1}{r_{4}} \dot{\tilde{k}}_{2} \tilde{k}_{2} .
$$

Let:

$$
\begin{aligned}
\dot{s}+\lambda s_{\Delta}= & \frac{1}{b}\left(\tilde{a} z_{2}+\tilde{b} u_{c}-\left(\hat{k}_{1}+\hat{k}_{2}\left|z_{2}\right|\right) \operatorname{sgn}\left(s_{\Delta}\right)\right. \\
& \left.+F_{l}^{\prime}+F_{f}^{\prime}+F_{w}^{\prime}+F_{g}^{\prime}\right) .
\end{aligned}
$$

Substituting Eq. 25) into Eq. 24, it follows that

$$
\begin{aligned}
\dot{V}(t)= & -b \lambda s_{\Delta}^{2}+\left(\tilde{a} z_{2}+\tilde{b} u_{c}-\left(\hat{k}_{1}+\hat{k}_{2}\left|z_{2}\right|\right) \operatorname{sgn}\left(s_{\Delta}\right)\right. \\
& \left.+F_{l}^{\prime}+F_{f}^{\prime}+F_{w}^{\prime}+F_{g}^{\prime}\right) s_{\Delta}+\frac{1}{r_{1}} \dot{\tilde{g}} \tilde{g}+\frac{1}{r_{2}} \dot{\tilde{h}} \tilde{h} \\
& \left.+\frac{1}{r_{3}} \dot{\tilde{k}}_{1} \tilde{k}_{1}+\frac{1}{r_{4}} \dot{\tilde{k}}_{2} \tilde{k}_{2}\right] \\
\leq & -\lambda b s_{\Delta}^{2} .
\end{aligned}
$$

With $V(t)-\lambda b s_{\Delta}^{2}$ and $V(t)$ is negative definite. Therefore, the tracking error $e(t)$ and the velocity of the tracking error, $\dot{e}(t)$ converge to zero.

\section{Controller Design of the Transportation Mo- bile Robot with the FTL}

The transportation robot is a consist of four main parts: left wheel, right wheel, lifter and FTE. Each part is actuated by using brushless DC motors. We designed each controller to control the position and velocity based on input values of the sensors and command inputs from the server. The encoder sensor gives noisy angular data of the motors rotation. Thus, a Kalman filter is used be decrease the sensor data noise. PID controllers are designed to control the velocity of each wheel of the mobile robot, as shown in Figure 7.

The model of the lifter and FTE has varying forces. Therefore, we apply a sliding mode and adaptive control method. The adaptive controller shown in Figure 7 is designed to control the velocity of the lifter and FTE smoothly and stably. We assume that the encoder sensor is continuously measured and the desired wheel positions are compared to stop the robot when it matches with the goal position on the path. A similar control structure is used for the FTL and FTE because the FTL and FTE have only different desired positions on the $\mathrm{z}$-axis and $\mathrm{x}$-axis path. 


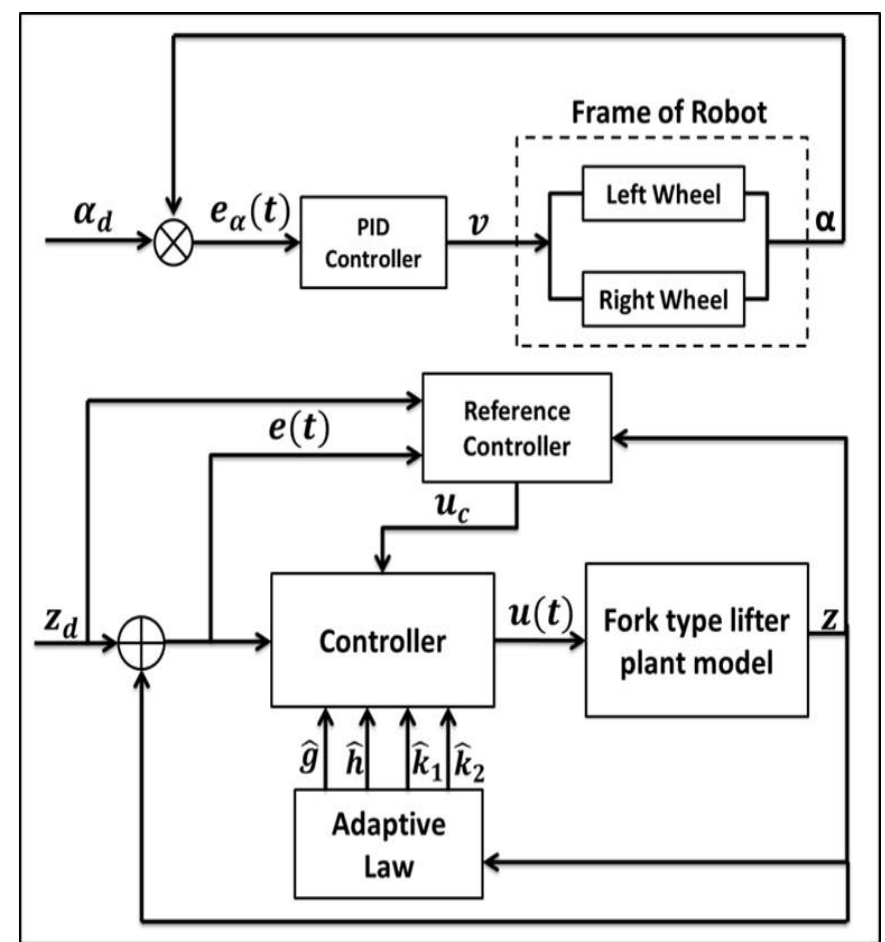

Figure 7. Overall control block diagram for the transportation mobile robot with FTL.

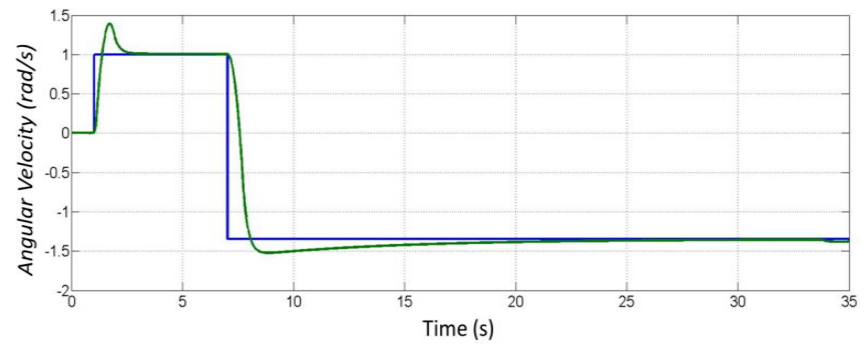

Figure 8. Output response of angular velocity with the PID controller.

\section{Simulation Results}

The MATLAB Simulink environment is used to evaluate the proposed control method. The kinematic and dynamic model of the transportation mobile robot with the FTL is used for the simulation. We assume that the wheel radius $R$ is $50 \mathrm{~mm}$.

The simulation result shows that the PID controller can stably track the desired angular velocity of the wheel as shown in Figure 8.

The dynamic model of the FTL is also used to evaluate the performance of the proposed adaptive controller. The parameters of the linear motor model are shown in Table 2. The output responses of the FTL system with a PID controller and
Table 2. Parameters of the linear motor

\begin{tabular}{ccc}
\hline Contents & Units & Values \\
\hline Force constant $\left(K_{f}\right)$ & $\mathrm{N} / \mathrm{A}$ & 8.5 \\
Resistance $(R)$ & $\Omega$ & 1.5 \\
Back EMF $\left(K_{e}\right)$ & $\mathrm{V} / \mathrm{m} / \mathrm{s}$ & 12.5 \\
Slide weight $(m)$ & $\mathrm{kg}$ & 15.0 \\
Armature inductance $(L)$ & $\mathrm{mH}$ & 2.2 \\
Peak current $\left(I_{p}\right)$ & $\mathrm{A}$ & 20.0 \\
\hline
\end{tabular}

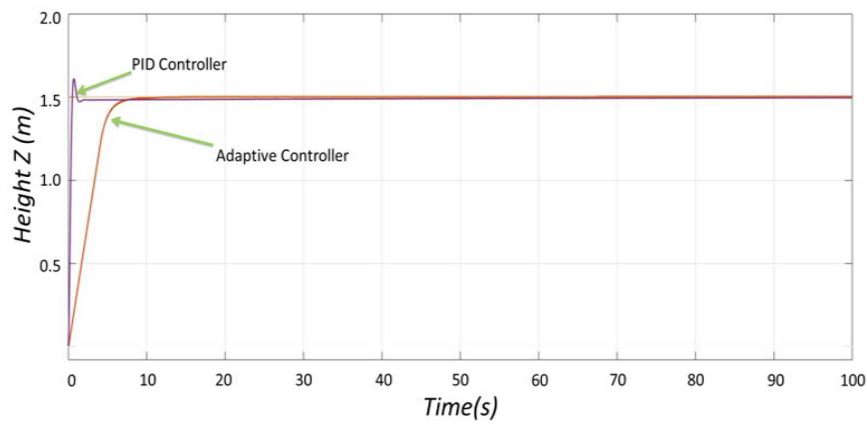

Figure 9. Output response of the FTL system with the PID controller and the proposed adaptive controller.

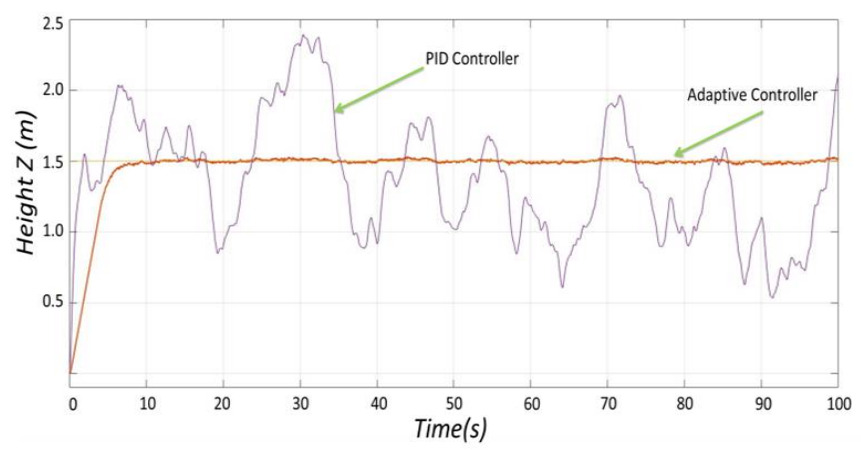

Figure 10. Output response of the FTL system with the PID controller and the proposed adaptive controller when the system has an external disturbance.

the proposed adaptive controller are shown in Figures 9 and 10. The simulation results show that the proposed adaptive controller can adapt with the sliding mode of the lifter quickly and smoothly.

\section{Conclusion}

This paper describes a controller design method for a transportation mobile robot with an FTL. PID controllers and an adaptive 
controller are developed to control the velocity of the wheels and the position of the FTL. The simulation results show that the transportation robot can follow the tracking path with desired velocity exactly and quickly. The robot can reach the package smoothly with the FTL system. In the future, we will apply the proposed control method to a real transportation mobile robot in a logistics center environment.

\section{Conflict of Interest}

No potential conflict of interest relevant to this article was reported.

\section{Acknowledgements}

This work was supported by the National Research Foundation of Korea (NRF) grant funded by the Korea Ministry of Education (No. 2015R1D1A1A01060400).

\section{References}

[1] Y. M. Kim and Y. T. Kim, "Design of logistics transportation robot based on modular conveyor rack and path planning in logistics center," Journal of Institute of Control, Robotics and Systems, vol. 22, no. 2, pp. 83-88, 2016. http://dx.doi.org/10.5302/J.ICROS.2016.15.0194

[2] N. T. Truc, E. H. Sun, Y. M. Kim, and Y. T. Kim, "Navigation method using fuzzy line tracking for the transportation robot," in Proceedings of 15th International Symposium on Soft Computing and Intelligent Systems (SCIS), 2014 Joint 7th International Conference on and Advanced Intelligent Systems, Kitakyushu, Japan, 2014, pp. 819-822. http://dx.doi.org/10.1109/SCIS-ISIS.2014.7044874

[3] N. V. Vui and Y. T. Kim, "Controller design for the lifting system of transportation robot," Proceedings of KIIS Spring Conference, vol. 26, no. 1, pp. 77-78, 2016.

[4] D. H. Jeong, J. I. Park, and Y. T. Kim, "Study on design of mobile robot for autonomous freight transportation," Journal of Korean Institute of Intelligent Systems, vol. 23, no. 3, pp. 202-207, 2013. http://dx.doi.org/10.5391/JKIIS. 2013.23.3.202

[5] P. F. Muir and C. P. Neuman, "Kinematic modeling of wheeled mobile robots," Journal of Field Robotics, vol. 4, no. 2, pp. 281-340, 1987. http://dx.doi.org/10.1002/rob. 4620040209
[6] A. Bara and S. Dale, "Dynamic modeling and stabilization of wheeled mobile robot," in Proceedings of the 5th WSEAS International Conference on Dynamical Systems and Control, Stevens Point, WI, 2009, pp. 87-92.

[7] F. A. Salem, "Dynamic and kinematic models and control for differential drive mobile robots," International Journal of Current Engineering and Technology, vol. 3, no. 2, pp. 253-263, 2013.

[8] M. Deng, A. Inoue, K. Sekiguchi, and L. Jiang, "Twowheeled mobile robot motion control in dynamic environments," Robotics and Computer-Integrated Manufacturing, vol. 26, no. 3, pp. 268-272, 2010. http://dx.doi.org/10. 1016/j.rcim.2009.11.005

[9] J. L. Avendano-Juarez, V. M. Hernandez-Guzman, and R. Silva-Ortigoza, "Velocity and current inner loops in a wheeled mobile robot," Advanced Robotics, vol. 24, no. 8-9, pp. 1385-1404, 2010. http://dx.doi.org/10.1163/ 016918610X501480

[10] B. S. Park, S. J. Yoo, J. B. Park, and Y. H. Choi, "Adaptive neural sliding mode control of nonholonomic wheeled mobile robots with model uncertainty," IEEE Transactions on Control Systems Technology, vol. 17, no. 1, pp. 207214, 2009. http://dx.doi.org/10.1109/TCST.2008.922584

[11] J. Wang, Z. Qu, M. S. Obeng, and X. Wu, "Approximation based adaptive tracking control of uncertain nonholonomic mechanical systems," Control and Intelligent Systems, vol. 37, no. 4, pp. 204-211, 2009.

[12] C. Samson, "Velocity and torque feedback control of a nonholonomic cart," in Advanced Robot Control. Heidelberg: Springer, pp. 125-151, 1991. http://dx.doi.org/10. 1007/BFb0039269

[13] F. Mnif and A. S. Yahmadi, "Recursive backstepping stabilization of a wheeled mobile robot," Proceedings of the Institution of Mechanical Engineers, Part I: Journal of Systems and Control Engineering, vol. 219, no. 6, pp. 419429, 2005. http://dx.doi.org/10.1243/095965105X33536

[14] R. Fierro and F. L. Lewis, "Control of a nonholonomic mobile robot: backstepping kinematics into dynamics," in Proceedings of the 34th IEEE Conference on Decision and Control, New Orleans, LA, 1995, pp. 3805-3810. http://dx.doi.org/10.1109/CDC.1995.479190 


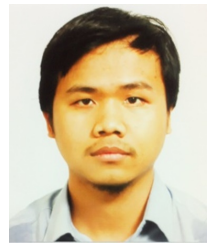

Nguyen Van Vui received his B.S. degree in Physics and Electronic Engineering, University of Science, Ho Chi Minh City, Vietnam in 2010. He was a firmware engineer at Greystone Data System, Ho Chi Minh City in 2010 and 2011. In 2011 and 2012, he was a hardware engineer at Elek Electronic, Binh Duong province. From 2013 to 2015, he was a software engineer in Robert Bosch Engineering Vietnam, Ho Chi Minh City. Since 2015, he has been working on a master's degree in the Department of Electrical, Electronic and Control Engineering at Hankyong National University, Anseong, Korea. His present interests include intelligent system and its applications.

E-mail: vuinguyen.qn@gmail.com

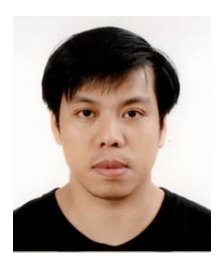

Tran Huu Luat received his B.S. degree in Physics and Electronic Engineering, University of Science, Ho Chi Minh City, Vietnam in 2010, and his M.S. degree in Electrical, Electronic and Control Engineering from Hankyong National University, Anseong, Korea, in 2014. From 2010 to 2012, he was a senior software programmer at Tli-Consulting Technology and Life Company, Ho Chi Minh
City, Vietnam. Currently, he is a Ph.D. candidate in Electrical, Electronic and Control Engineering from Hankyong National University. His current research interests include intelligent robots, automation, intelligent control and its applications.

E-mail: tranhuuluat@gmail.com

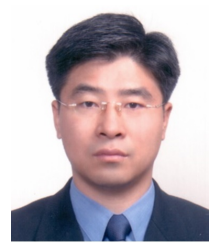

Yong-Tae Kim received his B.S. degree in Electronic Engineering from Yonsei University, Seoul, Korea, and his M.S. and Ph.D. degrees in Electrical and Electronic Engineering from KAIST, Daejeon, Korea, in 1991, 1993, and 1998, respectively. From 1998 to 2000, he was a senior engineering in Samsung. In 2006, he was a visiting scholar at Department of Electrical Engineering in University of Illinois at Urbana-Champaign. Since 2002, he has joined the faculties of the Department of Electrical, Electronic and Control Engineering at Hankyong National University, where he is currently a professor. His current research interests include intelligent robots, logistics automation, intelligent control and its applications.

E-mail: ytkim@hknu.ac.kr 